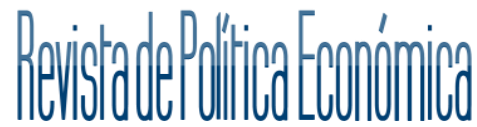

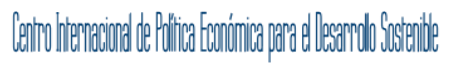

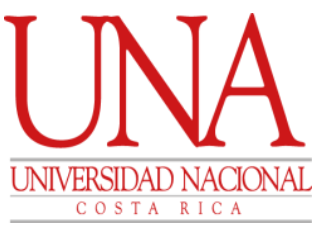

Revista de Política Económica para el Desarrollo Sostenible

Revista electrónica publicada por el Centro Internacional

de Política Económica para el desarrollo Sostenible

Universidad Nacional, Lagunilla, Heredia Apartado 2393-3000

\author{
Revista de Política Económica para el Desarrollo Sostenible \\ Revista Electrónica Semestral, ISSN-2215-4167 \\ Volumen 1, número 2 \\ Enero - Junio, 2016 \\ http://www.revistas.una.ac.cr/index.php/politicaeconomica
}

\section{Política fiscal y cambio climático en Costa Rica: oportunidades y desafíos}

Leiner Vargas Alfaro ${ }^{1}$

Fecha de recepción: 23.06.2015 Fecha de aceptación: 19.05.2016 Fecha de publicación:30.06.2016

\author{
(c) \\ Protegido bajo licencia Creative Commons \\ Universidad Nacional
}

\footnotetext{
1 Doctor en Economía. Catedrático de la Universidad Nacional. Docente del Centro Internacional de Política Económica para el Desarrollo Sostenible (CINPE) de la Universidad Nacional. leiner.vargas.alfaro@una.cr
} 


\title{
Política fiscal y cambio climático en Costa Rica: oportunidades y desafíos
}

\author{
Fiscal policy and climate change in Costa Rica: opportunities and challenges
}

Leiner Vargas Alfaro

\section{RESUMEN}

El presente artículo expone las distintas opciones, desafíos y posibilidades que tiene Costa Rica de alterar los incentivos en el campo fiscal con miras a reducir su impacto en el ambiente y abatir, a través de la reducción de emisiones y otras medidas, las consecuencias locales y globales del cambio climático. Este último y sus efectos se han convertido en uno de los principales problemas globales del siglo XXI. Si bien Costa Rica había comprometido una meta de carbono neutralidad al 2021, dicho proceso fue redefinido en la nueva estrategia al 2030. Costa Rica se ha propuesto convertirse en un país carbono neutro, lo cual no solo representa un reto en materia de reducción de las emisiones, sino que involucra un cambio tecnológico e institucional capaz de garantizar la sostenibilidad del propósito país. Identificar los actuales instrumentos fiscales y parafiscales, así como futuras posibles medidas en este campo es el enfoque metodológico utilizado, esencialmente a través de la información secundaria existente. Es claro que el país requiere de corregir los incentivos económicos de manera que se garantice propiciar tecnologías y procesos productivos bajos en emisiones. Para lo anterior, el uso de instrumentos fiscales puede ser de gran ayuda en el cumplimiento de este objetivo.

Palabras Clave: incentivo fiscal, instrumentos, medio ambiente, emisiones.

\section{ABSTRACT}

The author discusses the various options, challenges and possibilities that Costa Rica has to modify tax incentives in order to reduce their impact on the environment and abate the local and global consequences of climate change, by reducing emissions and taking other measures. Climate change and its effects have become one of the major global problems of the 21st. century. Although Costa Rica had committed to achieve the carbon neutrality goal by 2021, this process was redefined in the new strategy for 2030 . Costa Rica intends to become a carbon-neutral nation, which is not only a challenge in terms of reducing emissions, but also involves a technological and institutional change capable of ensuring the sustainability of the country's goal. Identifying current fiscal and quasi-fiscal instruments, as well as possible future measures in this field is the methodological approach used in this paper, mainly through the existing information. It is clear that the country needs to correct economic incentives to foster low-emission production processes and technologies. The use of tax instruments can be very helpful in achieving this objective.

Key Words: Technological electricity, regulatory framework, opening market 


\section{Introducción}

Costa Rica ha postergado por varias décadas una reforma fiscal integral; lo cual ha provocado fragilidad en el tema fiscal en el país. Dicha situación fiscal se ha agravado en los últimos años producto, entre otros temas, de la crisis internacional del periodo 20082009. De igual forma, las condiciones internacionales desfavorables para el crecimiento producto de la recuperación lenta de las economías occidentales y las crecientes presiones y desafíos del cambio climático y de los cambios en su estructura poblacional- ponen en el debate local la necesidad de reformar el sistema fiscal.

En este entorno es básico considerar no solo el objetivo hacendario, sino que podrían incluirse otros instrumentos que favorezcan la competitividad de la economía, la eficiencia y la sostenibilidad ambiental del país. A fin de proponer oportunidades y desafíos para la política fiscal y el cambio climático en Costa Rica, el presente artículo plantea, como objetivo central, identificar y discutir los instrumentos existentes tanto en el ámbito fiscal como parafiscal relacionados con el tema y realizar una valoración de estos en sus principales resultados. Para lograr dicho objetivo, el artículo se organiza de la siguiente manera.

En la primera sección se presenta una breve reseña de la situación fiscal en Costa Rica, analizando sus principales determinantes desde la perspectiva de los ingresos, el gasto, la deuda y su expresión en el déficit fiscal. La segunda y tercera sección se enfocan en desarrollar un análisis de los principales desafíos y oportunidades para integrar la equidad, sostenibilidad y competitividad del país, procurando una integración de las políticas públicas, coordinando así las decisiones en materia fiscal con las políticas ambientales y de cambio climático. El autor parte de la base de que la política de cambio climático debe ser un componente integral de la política pública en materia de ambiente y no debe analizarse de manera separada. Finalmente, el artículo cierra con una sección de conclusiones y recomendaciones, a través de un análisis de los posibles retos, medidas y desafíos institucionales para la integración de las políticas fiscales, ambientales y climáticas del país

\section{Situación fiscal de Costa Rica en materia fiscal}

Datos recientes del Ministerio de Hacienda para el 2015 dan cuenta de una proyección del déficit fiscal superior al 6 por ciento del Producto Interno Bruto (PIB). Además, los datos afirman que, de no realizarse ajustes, dicho déficit podría superar el 8 por ciento del PIB para el 2018 (Ministerio Hacienda, 2016). Siendo Costa Rica un país pequeño y abierto, su 
dinámica de crecimiento nacional depende fuertemente de la evolución de la economía mundial, particularmente sus socios comerciales principales: Estados Unidos, Centroamérica y el Caribe, y Europa. Si bien es posible en el corto plazo generar algunos estímulos a la demanda interna para crecer a mediano y largo plazo, la posibilidad de crecimiento económico sostenido y sostenible dependen de corregir las fallas estructurales de la economía nacional y de favorecer las condiciones para mejorar la competitividad internacional, lo que necesariamente requiere de inversión pública estratégica y de una mayor estabilidad fiscal (Organisation for Economic Co-operation and Development [OECD], 2016).

Es imposible un crecimiento sostenido sin un aumento en la productividad de los factores, lo que necesariamente nos lleva al tema de la educación, la salud, la mejora en la infraestructura física, el fortalecimiento de los indicadores tecnológicos y de inclusión digital; todo lo anterior requiere de una creciente participación del Estado y un tamaño que podría implicar entre 4 y 5 puntos porcentuales adicionales del PIB en gasto e inversión pública. No obstante, el país no puede aspirar a servicios públicos y bienes colectivos de primer mundo si no realiza ajustes importantes en los mecanismos de financiamiento de dichos bienes y servicios. En este sentido, una opción sería aumentar la carga fiscal y, por supuesto, corregir los excesos en algunos componentes del gasto actual, principalmente en incentivos salariales y pensiones.

En el gráfico 1 se puede observar cómo ha ido empeorando la situación del déficit fiscal del país. Después de haber estado en una situación de superávit durante dos años de la administración Arias-Sánchez (2006-2010), comienza un deterioro significativo, explicado tanto por una disminución de la carga tributaria al PIB, como por un aumento significativo del gasto primario. Más recientemente, la situación se ha vuelto más compleja al generarse nuevamente un aumento en el endeudamiento externo e interno de la economía, aumentando también el déficit secundario del Estado. Ante este creciente problema, surge la interrogante de ¿cómo enfrentarlo?, ¿a través de qué mecanismos, políticas o acciones? Así, por ejemplo, se considera la reducción del gasto público, el aumento de impuestos, o bien, una combinación de políticas alternativas que permita disminuir esta tendencia creciente del déficit de las finanzas públicas. 


\section{Gráfico 1.}

Costa Rica: Déficit fiscal como porcentaje del PIB datos observados y proyecciones (2006 -2015)

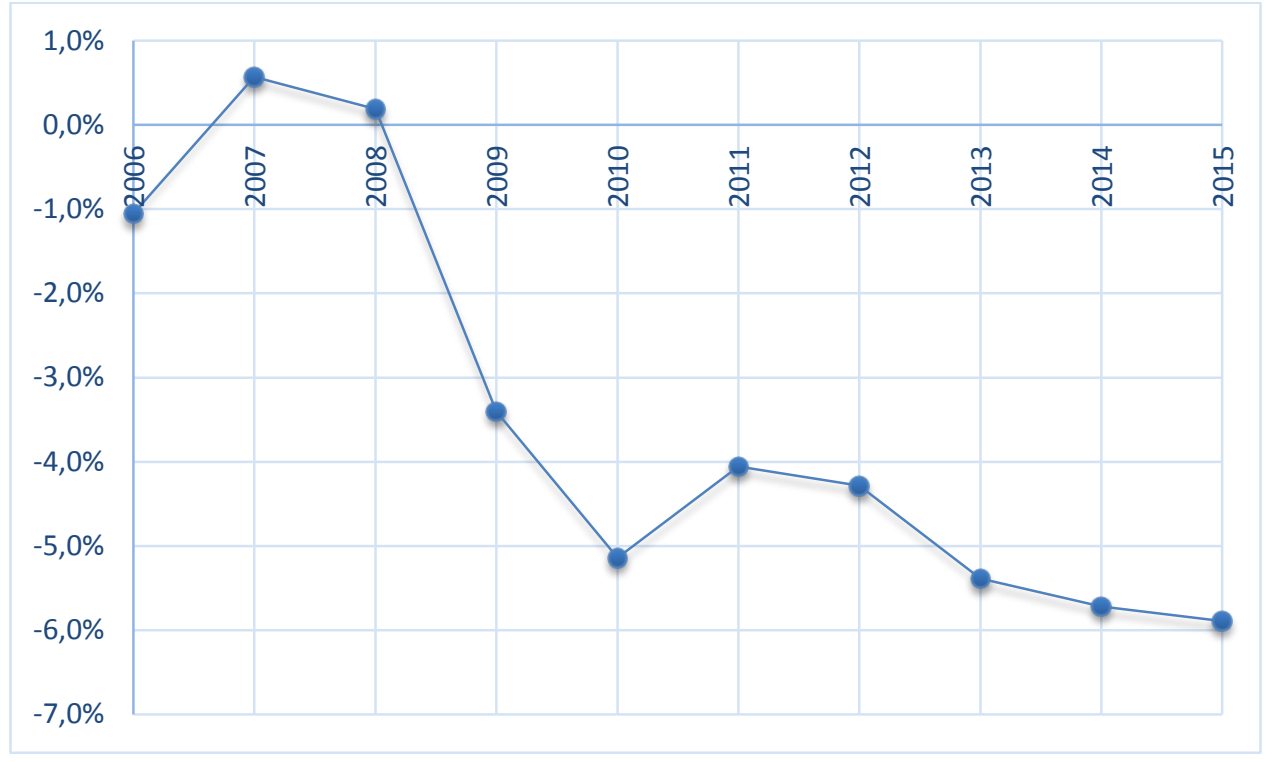

Fuente: Elaboración propia con datos del Ministerio de Hacienda (2016)

Es reconocido el esfuerzo que el gobierno de la administración Chinchilla Miranda 20102014 realizó por avanzar en la aprobación de la reforma fiscal. Sin embargo, a pesar de su aprobación por el Parlamento, la Sala IV o Sala Constitucional descartó la última versión del Plan Fiscal por encontrarle vicios al procedimiento en su aprobación en la Asamblea Legislativa (Ministerio de Hacienda,2013). En la segunda parte de la administración Chinchilla Miranda el esfuerzo siguió en la dirección de contener el gasto y de realizar una gestión de la deuda que permitiera al país disminuir el costo y la presión de la deuda sobre los mercados de dinero a nivel interno, aspecto que se logró gracias al uso de alrededor de 4000 mil millones de dólares en eurobonos, los cuales fueron un puente financiero en el caso de la administración actual, Solís Rivera (2014-2018). Esta tendencia al endeudamiento puede verse en la gráfica 2 a continuación, que muestra claramente un agravamiento de la condición fiscal de largo plazo del país. 
Gráfico 2.

Costa Rica: Deuda Pública del Gobierno Central como porcentaje del PIB (2000-2016*)

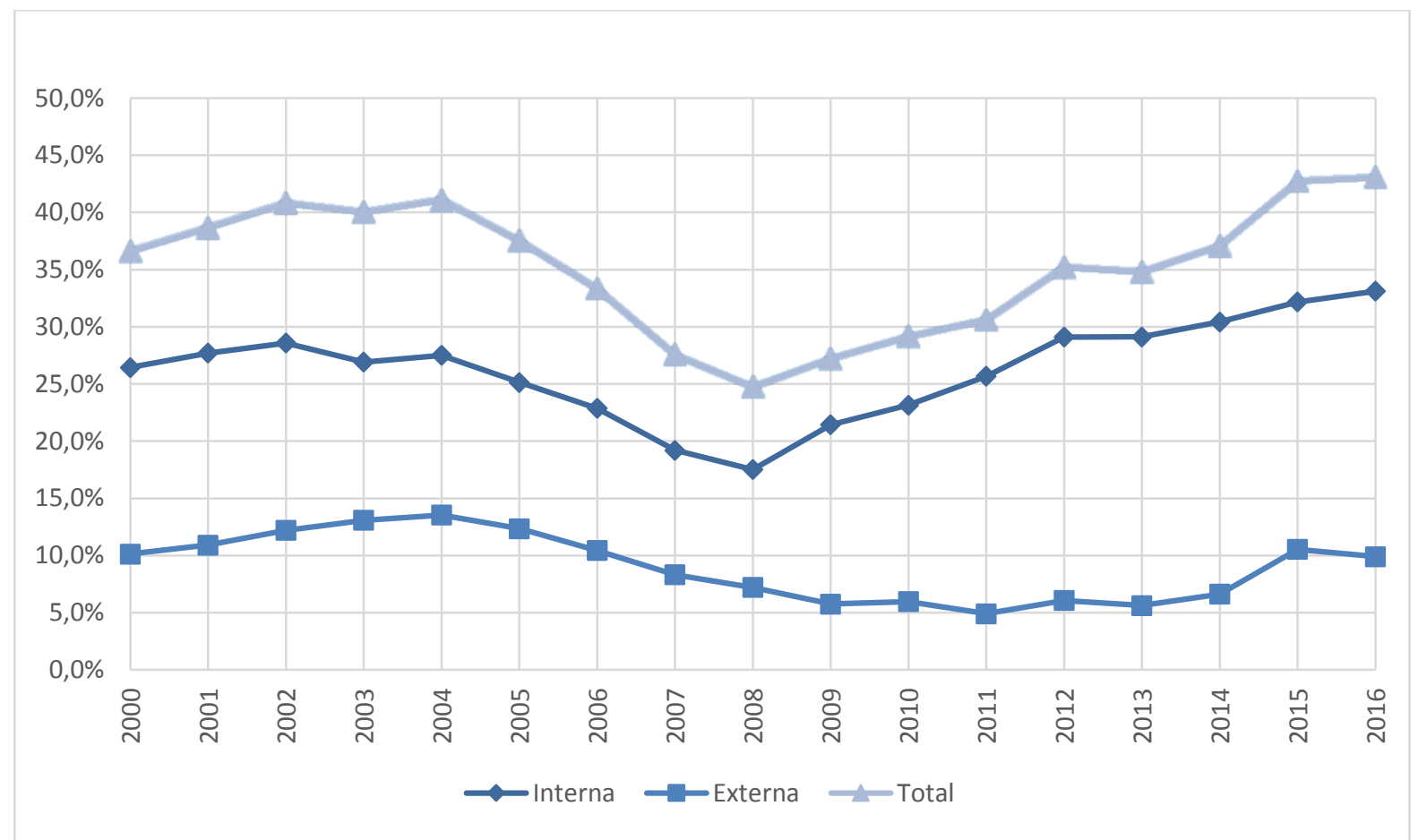

$\left(^{*}\right)$ Datos anuales de diciembre 2000 a diciembre 2015. El dato del 2016 corresponde al acumulado a abril

Fuente: Elaboración propia con datos del Ministerio de Hacienda (2016)

Muy a pesar de los esfuerzos por contener el gasto y mejorar la recaudación que se han hecho hasta el 2015, la situación fiscal del país sigue siendo crítica y podría pasar factura en un futuro cercano, de no realizarse medidas sustantivas en ambos componentes, gasto e ingresos (Fondo Monetario Internacional [FMI], 2016). Empero, se puede concluir que los desafíos fiscales en materia de gasto y de ajuste tributario siguen estando presentes en la agenda macroeconómica del país. Aunque si bien representan una amenaza, son al mismo tiempo una oportunidad para reasignar los incentivos de largo plazo, sobre todo en temas como el ambiente y, en particular, el cambio climático. 


\section{El cambio climático: Oportunidades para integrar la equidad, la sostenibilidad y la competitividad del país}

El cambio climático es un problema global que tiene grandes implicaciones a nivel nacional, regional y local en Costa Rica (Reino Unido Department for International Development, SIECA, DANIDA, NU. CEPAL. Subsede de México y Comisión Centroamericana de Ambiente y Desarrollo, 2012). Es por eso que urge la necesidad de tomar decisiones en el ámbito de las políticas públicas. Empero, es esencial también que dichas políticas mantengan un balance adecuado entre el bienestar de la población y la sostenibilidad de los ecosistemas. Costa Rica se ha propuesto el ambicioso objetivo de la carbono neutralidad 2021, que si bien recientemente ha sido modificado con un nuevo acuerdo y ofrecimiento ante la Convención sobre el Clima de Naciones Unidas por parte del Ministerio de Ambiente y Energía, MINAE (Paniagua, 2016), el tema sigue significando un gran desafío por enfrentar en la siguiente década. Es clara entonces la necesidad de tener un eje de política pública dirigido al diseño y la definición de acciones e instrumentos pertinentes, coherentes y articulados que reduzcan las emisiones y favorezcan una adecuada adaptación de la sociedad a los efectos del cambio climático (OECD, 2016).

A nivel internacional, Costa Rica es reconocido como un país ejemplar en materia de políticas de conservación y protección de la biodiversidad (PNUMA-GEO-5, 2012). Sus contribuciones van desde el aseguramiento en zonas de protección absoluta y relativa de una porción equivalente a la cuarta parte del territorio nacional, hasta el más reciente compromiso del Estado por conseguir convertirse en el primer país carbono neutral del mundo en el año 2021. Este compromiso, si bien ha sido modificado por la presente administración Solís Rivera (2014-2018), continúa siendo relevante en el ámbito privado y de accionar de la sociedad. Sin embargo, ante las limitaciones fiscales del Estado, muchas veces se dejan de lado los grandes desafíos en materia de desarrollo sostenible y calidad ambiental, privilegiándose los objetivos de corto plazo de asegurar ingresos o de no afectar la competitividad de ciertas actividades económicas y actores de la sociedad (Fanelli, J.M, Jiménez, J.P y López, I; 2016).

Los principales temas asociados con el medio ambiente en general y el cambio climático que son objeto de interés para la política fiscal o parafiscal verde son, en particular: la preservación, el ahorro y el uso eficiente del recurso hídrico; la contaminación y la gestión de residuos sólidos; el ordenamiento territorial y espacial; la producción sostenible agrícola -que involucra la disminución de uso de pesticidas- y, por supuesto, el uso racional de energía y su producción a base de recursos renovables limpios, el transporte y la gestión 
ambiental (Jiménez, Amit y Vindas, 2010). Todo lo anterior puede verse, en forma sistémica, como parte de la agenda país de cara a integrar la política fiscal, gastos e ingresos, dentro de la protección y uso sostenible de los recursos naturales, entre ellos, los que se refieren al clima².

Dada la situación actual en materia fiscal y la agenda integral de política ambiental y de clima, el posible programa de impuestos verdes o de incentivos a la economía baja en carbono -tarifas fiscales y parafiscales verdes- podría ser visto, entonces, como una oportunidad histórica de fortalecer la estructura recaudatoria y, al mismo tiempo, favorecer instrumentos que permitan incentivos a una producción más limpia y una menor intensidad en el uso del carbono y de contaminantes en general. Este desafío del país de integrar la política fiscal con el medio ambiente es esencial para fortalecer los vínculos entre los incentivos económicos de corto plazo y la visión país a largo plazo, que pretende ser una economía baja en emisiones y capaz de adaptarse ante el cambio climático global.

\subsection{Coordinación de las políticas públicas: Oportunidades y desafíos para integrar la política fiscal y el medio ambiente}

Tal y como se plantea en los planes de desarrollo de las últimas dos administraciones (Ministerio de Planificación Económica y Política Económica [MIDEPLAN], 2010; MIDEPLAN, 2015) en el eje ambiental de Costa Rica, se precisa de plasmar una serie de propuestas en pro de resguardar el patrimonio natural sin dejar a un lado la meta de crecimiento económico. A lo anterior se puede denominar como un crecimiento sostenible. Para lograrlo, es necesario promover y sobreponer la meta de la carbono neutralidad, esto se puede complementar según a lo planteado, con un ordenamiento territorial que incorpore de manera efectiva la variable ambiental, el uso racional de los recursos, el fomento de energías limpias y, por supuesto, la protección de los ecosistemas vulnerables y del recurso hídrico. En términos de política fiscal, existe un gran desafío a nivel nacional en cuanto a la integración de las políticas y su impacto ambiental.

Es notable la gran distancia existente entre las aspiraciones de los gobiernos y de los tomadores de decisiones y los logros obtenidos en temas de desarrollo sostenible: "Muchas de las políticas públicas existentes y sobre todo, los instrumentos fiscales pueden por el contrario ser perversos respecto al medio ambiente, promoviendo usos insostenibles de diversos bienes o fomentando matrices de producción que afectan y aumentan las emisiones" (CEPAL, 2016). En el diseño de la política fiscal, se le debe dar relevancia al

\footnotetext{
${ }^{2}$ Es importante distinguir entre la discusión estrecha de impuestos verdes y la dimensión más amplia de la fiscalidad y el desarrollo sostenible.
} 
desarrollo sostenible, tomando en cuenta los efectos que dichas políticas fiscales tienen en áreas como la económica, el ambiente y las condiciones sociales, entre otras. Esta integración es de gran ayuda para alcanzar un desarrollo sostenible a largo plazo, de manera que se logre satisfacer las necesidades presentes sin comprometer los recursos de las generaciones futuras.

Es también prudente valorar las exoneraciones fiscales y las medidas de gasto 0 inversión, en sus efectos meritorios o perversos respecto al medio ambiente, buscando generar un equilibrio entre las prioridades de financiamiento del gasto ${ }^{3}$ e inversión del Estado y los efectos de dicho gasto e ingresos en la sostenibilidad de los ecosistemas y los recursos naturales en general. La falta de coordinación presente al interior de la definición de las políticas públicas genera que, no en pocas ocasiones, las políticas adoptadas por un lado dan una solución paliativa a uno de los problemas que se desea combatir, pero por otro lado agravan en una proporción mucho mayor a otros sectores.

Esto conlleva a la generación de externalidades negativas por parte de las mismas políticas públicas, las cuales muchas veces son innecesarias y pueden ser contrarrestadas con una eficiente integración de las políticas. En la siguiente sección se mostrará algunos ejemplos de este tipo de situaciones que afectan claramente el accionar y los incentivos a favor del ambiente para Costa Rica (Orozco y Ruiz (2001); CEPAL, 2010). Por ello, es necesario poner un énfasis especial en la obligación por mejorar la integración y gestión de las políticas fiscales y los objetivos ambientales y de cambio climático, haciendo hincapié en aquellas que tienen la capacidad de influir de manera directa en las metas nacionales.

\subsection{Impuestos verdes y reforma fiscal ambiental}

Existe una tendencia creciente en el contexto internacional por la implementación de impuestos verdes o ambientales 3 y se ha procurado incluir estos como parte de una reforma fiscal integral, principalmente en los países más desarrollados (Acquatella y Gómez, 2005). Costa Rica, dado su objetivo de convertirse en un país carbono neutral, debe preocuparse por potenciar la implementación de impuestos verdes, de esta manera se lograría integrar un régimen fiscal en concordancia con los objetivos de desarrollo sostenible. Según la CEPAL (2016), el posible papel de los impuestos ambientales dentro del ámbito fiscal son:

- Instrumentos para internalizar el costo social de actividades económicas que conllevan impactos ambientales, creando así una estructura de incentivos

\footnotetext{
${ }^{3}$ Se entiende por impuesto verde o medida ambiental verde aquellas que propician o favorecen las decisiones de consumo o producción ambientalmente favorables. También se puede ampliar el margen a reformas fiscales ambientales como aquellas que integralmente miran el medio ambiente y la protección de los recursos naturales como principio básico, no solo del lado de los impuestos pero también en relación con el gasto. Se incluyen de estos por ejemplo las medidas asociadas a las compras verdes, aduanas verdes y otras acciones originadas en la hacienda pública que pueden favorecer el vínculo sistémico con los ecosistemas naturales (Vargas, 2014).
} 
compatibles para reducir la degradación ambiental a través del sistema de precios.

- La utilización de impuestos ambientales, tasas y cargos por el uso de recursos naturales y el medio ambiente, con fines recaudatorios en general 0 de contención en el consumo de ellos.

- El diseño del régimen impositivo que aplica a la explotación de recursos naturales para inducir una utilización acorde con los objetivos nacionales de desarrollo sostenible y de gestión integral de los recursos y el medio ambiente.

Si bien los impuestos o subsidios pueden llevar importantes señales en los precios para proteger el ambiente, más recientemente se han encontrado formas distintas de fomentar una fiscalidad verde. Se trata de medidas por el lado del gasto y formas alternativas de promover lo que, se podría decir, son bienes y servicios ambientalmente amigables. De igual manera, es urgente corregir los subsidios o exoneraciones que por otros fines se han instalado y verificar el nivel de impacto ambiental que dichas exoneraciones tienen. De una u otra forma, se trata de una valoración global y no simplemente del esquema de impuesto verde clásico.

\subsection{Situación de Costa Rica en materia de impuestos y tarifas cuasifiscales verdes}

Si bien Costa Rica tiene un reconocimiento global por la protección del medio ambiente, el tema fiscal es aún poco utilizado para promover incentivos de comportamiento que favorezcan el desarrollo sostenible. Solamente existen una limitada cantidad de impuestos relacionados con este tema. Entre otros elementos, esto ha causado que no se logre producir cambios realmente significativos al desarrollo sostenible en diversas agendas, como por ejemplo el manejo de residuos sólidos, el uso del agua, el ahorro de energía eléctrica y combustibles, entre otros.

De acuerdo con González y Rodríguez (2008), "es posible aprender de las experiencias de otros países en la implementación de mecanismos fiscales para la gestión de soluciones ecológicas, de manera que se adapten a las características de un país en vías de desarrollo, como Costa Rica". Dado lo anterior, en el presente apartado se hace una descripción breve de las principales acciones implementadas en el país en tema de impuestos verdes y tarifas cuasifiscales orientadas al medio ambiente.

\section{- Pago por Servicios Ambientales}

Dentro de los principales aportes de Costa Rica en el tema de impuestos verdes se encuentra el referente al Sistema de Pago por Servicios Ambientales (SPSA). Si bien la designación original del impuesto a los combustibles no fue vista 
como parte de una reforma fiscal verde, un componente del mismo fue orientado específicamente a la protección del bosque y, en particular, a financiar el SPSA. Este se crea bajo la necesidad especial permitida por ley para compensar el daño del medio ambiente ocasionado como respuesta de la deforestación y el cambio en el uso de la tierra. Actualmente, el financiamiento de este sistema está compuesto en buena parte por la recaudación proveniente del impuesto único a los combustibles, fondos externos de cooperación y mecanismos voluntarios entre empresas y el Estado. Es notable también la aprobación reciente de una tarifa hídrica en la ARESEP para este mismo fin, que permite un fondo creado y cobrado en el recibo de agua de las empresas, incluyendo el principal proveedor del servicio, Acueductos y Alcantarillados (AYA).

Este instrumento ha sido descrito por varios autores como una de las principales ecoinnovaciones en el mundo. Para el país, ha significado una clara reducción y revertimiento de la situación en materia forestal, favoreciendo un aumento en la cobertura y una mayor sostenibilidad de los bosques (Segura, 2000).

Según el informe Estado de la Nación (2015), en su capítulo ambiental, el pago por servicios ambientales evitó la deforestación y aumentó el bienestar de las familias que viven en los alrededores de los parques nacionales. Es claro el impacto que este tipo de instrumentos ha tenido en favorecer la reforestación y sostener la biodiversidad, ayudando también a la recarga acuífera y contribuyendo a mejorar la hidroestacionalidad y la escorrentía, ambas vitales para la industria eléctrica basada en empresas hidroeléctricas.

\section{- Canon de aprovechamiento de Aguas}

Otra de las medidas ambientales aplicadas en Costa Rica es el canon de aprovechamiento de aguas. Dicho instrumento económico existe para la regulación del aprovechamiento y la gestión del recurso hídrico. Con este, se espera contar con una disponibilidad hídrica para un abastecimiento estable tanto para consumo doméstico como para las actividades que promueven el desarrollo socioeconómico del país. Además, busca la generación de recursos económicos con el objetivo de financiar a largo plazo una gestión sostenible del agua.

Este canon es cobrado a todos los ciudadanos del país, sin exclusión por ser persona física o jurídica. Su aplicación sigue una lógica diferenciada según el tipo de consumo, sea este doméstico, industrial, agropecuario, turístico, entre otros. Según el decreto Ejecutivo N. 32868 del 2005, este canon contempla el valor agregado de las aguas subterráneas, los costos de administración y gestión del recurso, el monitoreo hidrológico y meteorológico, además de los costos de manutención, conservación y recuperación de las cuencas hidrográficas. 
Su tarifa se calcula con base en el volumen de consumo, utilizando como unidad de medida los metros cúbicos. Dicha tarifa incluye una reducción con el fin de incentivar la producción de energías limpias a partir del uso de este recurso. Aproximadamente el $50 \%$ de los ingresos obtenidos a través de este sistema se dirigen a una gestión de desarrollo con la finalidad de conseguir los siguientes objetivos: un uso sostenible del agua, una incorporación de recursos tecnológicos para la gestión del recurso, monitoreo meteorológico e hidrológico, garantizar una gestión hídrica en todo el territorio nacional, desarrollo de infraestructura de aprovechamiento para el manejo eficiente de la demanda y oferta de agua, además del financiamiento de proyectos y acciones destinadas a la conservación, restauración, uso sostenible y protección de las cuencas hidrográficas y sus recursos. El 50 \% restante se utiliza para la reinversión en la cuenca generadora de este recurso con el fin de mantener y recuperar los ecosistemas aledaños.

\section{- Canon de vertidos}

El canon de vertidos sobresale como un instrumento de regulación, ya que se crea con la finalidad de alcanzar un medio ambiente ecológicamente equilibrado a través de la internalización de las externalidades causadas por los vertederos.

Rodríguez y Echeverría (2003) lo describen de la siguiente forma: "El servicio ambiental que presta el agua a quienes la usan para el vertimiento; y el costo social y ambiental que dicho uso implica" (p. 12). El servicio ambiental que proporciona este recurso es lo que se toma de fundamento para el establecimiento de este canon.

El dinero recaudado mediante esta vía tiene como destino la cuenca hidrográfica de origen. Se establece que solo se pueden financiar o invertir en proyectos de alcantarillado sanitario, sistemas de tratamiento de aguas residuales municipales, proyectos de investigación que promuevan el desarrollo de procesos productivos y tecnológicos que se orienten hacia un mejor y más eficiente uso de los recursos hídricos.

\section{- Impuesto único sobre los combustibles}

Este impuesto se dirige principalmente a grabar la producción nacional, la cual incluye la destilación y refinería del combustible, así como la importación de combustibles en el momento de la aceptación aduanera en todo el territorio nacional. La base imponible de este impuesto se estima sobre un monto fijo por litro, diferenciando según el tipo de combustible. De este impuesto se exoneran las misiones diplomáticas y los organismos internacionales, además del producto 
destinado a abastecer líneas áreas, buques mercantiles o de pasajeros con líneas comerciales, la Cruz Roja Costarricense y la flota de pescadores nacionales.

Según la regulación nacional establecida en la Ley N.8114, se debe trasladar el 3,5\% de la recaudación de este impuesto para el FONAFIFO, con el propósito de cumplir con los pagos por servicios ambientales.

Este impuesto parece revestir las características de los tributos medioambientales, pues además de que su recaudación se encuentra destinada a fines medioambientales, la ley que lo crea deja de sujetar los combustibles al pago de los siguientes impuestos: impuesto selectivo de consumo, impuesto correspondiente al $1 \%$ sobre el valor aduanero de las importaciones creado por la Ley No. 6946, impuesto general sobre las ventas y otros derechos arancelarios a la importación" (González y Rodríguez, 2008).

- Impuesto sobre la propiedad de vehículos automotores, embarcaciones y aeronaves

Este impuesto busca grabar en todo el territorio nacional los automotores inscritos en el Registro Público de Vehículos, además de todas las aeronaves registradas debidamente en el Registro de Aviación Civil y las embarcaciones registradas en la Dirección General de Transporte Marítimo. Esta recaudación tiene origen en el momento de la adquisición del bien y se mantiene hasta la cancelación de la inscripción del mismo.

Su base imponible se calcula a enero de cada año, según el valor del bien en el mercado interno, esto para el caso de los vehículos automotores; para las embarcaciones y vehículos aéreos, su base impositiva se fija según el decreto ejecutivo emitido anualmente. Las tarifas de este impuesto son de carácter progresivas y varían entre un 1,2\% y un 3,5\%.

El mercado nacional tiene una elevada presencia de vehículos usados y esto sin duda recae en una evidente vulnerabilidad del país en temas de consecuencias ambientales, de salud y económicas. Sin embargo, esta situación tiene algunas implicaciones de carácter fiscal. Pese a la inexistencia de estudios significativos acerca de la posible influencia que dicha situación pueda tener en la calidad de vida de los costarricenses y en nuestro entorno natural, lo cierto es que el hecho de que la mayoría de los automóviles en el país tengan más de 5 años desde su fabricación tiene una incidencia casi directa en la calidad del aire, en virtud del aumento de la cantidad de emisiones atmosféricas de monóxido de carbono que los vehículos usados emanan. 
A pesar de esta problemática y en contraste con los objetivos nacionales de conservación, la Presidencia, el Ministerio de Hacienda y el Ministerio de Economía emitieron un decreto ejecutivo que establece una rebaja del impuesto selectivo de consumo a los autos usados. Lo anterior es una clara muestra de la incoherencia en las políticas públicas nacionales, en este caso en lo referente al tema de la fiscalidad ambiental. Esta incoherencia va en contra del objetivo primordial de este impuesto, el cual consiste en incentivar la adquisición de automóviles más nuevos que consuman menos combustible y por ende causen un menor daño al ambiente.

Según la tarifa actual, los autos nuevos y usados de modelos hasta tres años anteriores deben pagar un tributo equivalente al $30 \%$ de impuesto selectivo de consumo, los usados de modelos de cuatro o cinco años anteriores deben pagar un $40 \%$ y los usados de modelos de seis o más años anteriores deben cancelar el 53 $\%$. Con la nueva propuesta del Ministerio de Hacienda, los autos nuevos y usados de modelos de hasta seis años anteriores enfrentarán un impuesto selectivo de consumo de $30 \%$ y tendrán una carga impositiva total de $53 \%$, mientras que los usados de modelos de siete o más años anteriores, una tarifa de $48 \%$.

Este tipo de impuestos sobre los vehículos, en general, se puede considerar verde en tanto inhibe la compra de autos individuales y favorece una mayor elasticidad y gasto en transporte público. Este es todo un nuevo ámbito que se encuentra en la palestra regulatoria, asociado con la movilidad y el uso de otros instrumentos para favorecer una disminución de la demanda efectiva de transporte y sus consecuencias sobre el ambiente en el país.

\section{Políticas ambientales, oportunidades y desafíos para la política fiscal}

Conocer el uso de las herramientas de política pública, en especial las que generan algún impacto en el área ambiental, se ha convertido en un factor elemental a la hora de tomar decisiones. Para ello, es necesario identificar los actores involucrados, el marco legal y regulatorio, el paquete de incentivos implícito en las políticas y, por supuesto, conocer los principales mecanismos o metodologías a utilizar. Con base en lo anterior, es indispensable identificar los posibles desafíos y oportunidades que implican la integración de estas políticas ambientales a las demás políticas públicas del país.

En específico, debe procurarse la integración de políticas de carácter fiscal con sus implicaciones en materia ambiental. Para el caso de Costa Rica, dado su compromiso de convertirse en un país carbono neural, se debe procurar una integración de las políticas de carácter macroeconómico y sectorial de manera que 
estas estén orientadas a alcanzar las metas nacionales de desarrollo sostenible. La existencia de impuestos ambientales ha sumado importancia en los últimos años y estos se han convertido en parte elemental de los planes de reforma fiscal en muchos países desarrollados. Costa Rica, a pesar de ser uno de los países con mayor desarrollo en la región en términos de impuestos ambientales, aún no logra alcanzar un nivel óptimo de coordinación de sus políticas que permitan solventar eficientemente los problemas de esta índole. La alta evasión, la deficiencia en la captación, las restricciones políticas e institucionales, entre otras, son solo algunas pocas de la amplia gama de limitantes a las que se enfrenta día a día el sistema tributario costarricense.

Es en este contexto es donde se identifica la necesidad de reformar la estructura tributaria nacional, aplicando el uso de impuestos verdes en distintos sectores claves que permitan reducir las emisiones contaminantes, de manera que se logre hacer más coherente las políticas fiscales aplicadas y que estas estén estrechamente ligadas a las metas ambientales del país. Una reforma fiscal eficiente y compatible con las metas ambientales debe ser aquella definida bajo un esquema de impuestos, basados en el principio de quién contamina paga. Por esta razón, es necesario establecer impuestos que se dirijan de forma más directa a las actividades contaminantes que las lleven hacia un nivel socialmente deseable, el cual incluya la totalidad de los costos sociales que implica el desarrollo de una determinada actividad económica.

Sectores como transporte, energía, agricultura y servicios son importantes generadores de externalidades ambientalmente negativas, lo que justifica la necesidad de la implementación de nuevos tributos o reformas en estos sectores que contribuyan a mejorar la calidad ambiental del país.

\subsection{Sector energía}

La matriz energética nacional está compuesta en un gran porcentaje por energía derivada del petróleo, con lo que se resalta la alta dependencia de esta fuente. Para el caso específico del subsector eléctrico, Costa Rica históricamente ha concentrado su matriz eléctrica en gran parte en la generación hidroeléctrica: en la actualidad, esta abarca casi el 80 \% del total de la producción nacional. Sin duda, esto ha sido un gran soporte a nuestra creciente demanda, mas no ha sido suficiente para abastecer por completo las necesidades del mercado local, principalmente por su elevada inestabilidad y sus picos de producción dependiendo de las distintas épocas del año. Como consecuencia de lo anterior, las empresas productoras, 
principalmente el ICE, productor principal y encargado de distribución a nivel nacional, se han visto ante la necesidad de utilizar plantas de generación térmica como plan de contingencia ante las posibles caídas de producción de las fuentes mayoritarias. A pesar de que su aporte es pequeño en relación con las demás fuentes, su impacto sobre el ambiente es grande, principalmente por la cantidad de emisiones peligrosas y contaminantes consecuentes de su producción.

El mercado eléctrico nacional debe preocuparse por buscar nuevas alternativas para el futuro. Para un país que busca ser carbono neutral en pocos años, la incorporación de nuevas formas de generación eléctrica amigables con el ambiente sería un buen comienzo para iniciar a incursionarse en este camino. No solamente se debe pensar en las tradicionales fuentes de generación, sino que se debe incorporar, como parte de un proceso de innovación, el ingreso de nuevas y pioneras tecnologías que vengan a complementar, o bien, por qué no, a sustituir en un futuro próximo las actuales fuentes de generación eléctrica con la finalidad de obtener una matriz energética más limpia, eliminando por completo la dependencia actual de los derivados del petróleo. Costa Rica requiere un avance rápido hacia una nueva matriz sostenible de energía que le permita continuar en la búsqueda de alcanzar un desarrollo sostenible y que a su vez mantenga su ventaja competitiva. De no darse lo anterior, se limitará el crecimiento económico y el bienestar social, lo cual provocaría consecuencias significativas para el ambiente y la salud. No cabe duda de que el conocimiento, de la mano con la tecnología, continúan siendo los impulsores clave del desarrollo económico sostenible, el avance social y la protección ambiental.

Costa Rica cuenta con una amplia gama de características que favorecen el desarrollo de energías limpias de fuentes renovables:

-Al ser un país montañoso con un elevado nivel de pluviosidad, tiene un amplio potencial para el desarrollo de proyectos de carácter hidroeléctrico.

-Cuenta con territorios ventosos en zonas donde el viento es consistente y fuerte, alcanzando velocidades de hasta $7 \mathrm{~m}$ por segundo, característica potenciadora para el desarrollo de proyectos eólicos. - La existencia de una cadena volcánica a lo largo de todo el país le permite potenciar la explotación de los recursos geotérmicos para la generación eléctrica. 
-Además, dada su ubicación geográfica, Costa Rica cuenta con una adecuada radiación y amplios terrenos agrícolas lo que favorece el desarrollo de proyectos solares y de biomasa.

Para lograr un cambio en el sector energía en nuestro país, es elemental enfocarse en dos aspectos centrales: la generación y la eficiencia, ambas orientadas hacia una generación más limpia haciendo uso mayoritariamente de fuentes renovables. Actualmente, un alto porcentaje de las inversiones en el sector energía son de carácter público, esto pone en evidencia la necesidad de mejorar los incentivos de entrada a empresas privadas que incorporen al país nuevas alternativas de generación limpia.

Además, dicho cambio requiere ir acompañado de políticas e incentivos que conviertan estas nuevas alternativas en opciones de inversión más atractivas. Esto lleva a una revisión de las metodologías tarifarias actuales, las cuales están basadas, principalmente, en un modelo de valoración de de activos financieros y no incorporan ninguna variable de carácter ambiental.

\subsubsection{Metodologías tarifarias, lejos del compromiso con el ambiente}

Actualmente, la Autoridad Reguladora de los Servicios Públicos (ARESEP) utiliza tres distintas metodologías para la definición de las tarifas eléctricas. En este aparatado se dará una breve explicación de cada una de ellas, con el propósito de identificar la necesidad de integrar el establecimiento de tarifas en Costa Rica del subsector eléctrico a partir de la preocupación por la conservación ambiental. A partir de esto, se concluye la necesidad de una integración del componente ambiental dentro de la estimación tarifaria (Barrantes, 2010).

A. Metodología de tasa de retorno. A través del uso de los ingresos y gastos derivados de la producción eléctrica, se obtiene como resultado la rentabilidad, la cual esta en función de la relación entre el ingreso neto de operación y una base tarifaria, tal y como se denota en la siguiente ecuación.

$$
R=\frac{I N O}{B T} \quad ; I N O=I-C O M A \quad ; B T=A F N O R+K T, \text { donde }:
$$


$\mathrm{INO}=$ ingreso neto de operación

$\mathrm{BT}=$ Base tributaria

COMA = Costos de operación, mantenimiento y administración

AFNOR=Activo fijo neto en operación revaluado

$\mathrm{KT}=$ Capital de trabajo

B. Promedio ponderado del costo del capital (WACC). Esta metodología busca definir un precio a la producción eléctrica a través de la definición del costo de capital de la empresa rk, donde este se define como:

$$
r_{k}=r_{d}(1-t) D / V+r_{k p} K P / V ; \text { donde: }
$$

$r_{k}=$ Costo de capital de la empresa

$r_{d}$ Costo del endeudamiento

rkp Costo del capital propio

$\mathrm{t}=$ Tasa impositiva

$\mathrm{D}=$ Valor de la deuda

$\mathrm{KP}=$ Valor del capital propio

$\mathrm{V}=$ Valor total de los activos $(\mathrm{D}+\mathrm{KP})$

C.Valoración de activos de capital (CAPM). Esta metodología incorpora, además de los costos de capital propio de la empresa, distintas variables de riesgo además de un parámetro $\beta$ que mide la correlación entre los rendimientos de mercado y los de una inversión específica, tal y como se muestra a continuación: 


$$
\begin{aligned}
& \quad r_{k p}=r_{l}+\beta\left(r_{m}-r_{l}\right)+r_{p} ; \text { donde: } \\
& r_{k p} \text { Costo del capital propio } \\
& r_{m}=\text { Rendimiento de una cartera diversificada de acciones } \\
& r_{l}=\text { Tasa libre de riesgo. } \\
& r_{m}-r_{l}=\text { Prima de riesgo. } \\
& r_{p}=\text { Riesgo país. } \\
& \beta=\text { Mide la correlación entre los rendimientos de mercado y los } \\
& \text { de una inversión específica. }
\end{aligned}
$$

Las metodologías tarifarias utilizadas por ARESEP, tal y como se denotó anteriormente, a pesar de ser eficientes para lograr el objetivo regulatorio, no contemplan ningún componente ambiental que garantice el uso y el manejo eficiente de los recursos naturales en los procesos productivos. Dado lo anterior, es importante la incorporación de un factor ambiental en el cálculo de las tarifas eléctricas, una variable que garantice la internalización de los efectos 0 externalidades negativas producidas en cada uno de los procesos y que a su vez incentive la inversión en energías más limpias, mejorando la calidad de la matriz eléctrica nacional e incentivando la entrada de nuevas tecnologías de generación eléctrica amigable con el ambiente. Un elemento importante a considerar en este aspecto es el mecanismo que se determinará para la recaudación y uso de este tipo de rentas cuasi-fiscales o tarifas adicionales ambientales. En este aspecto, se puede generar un fondo ambiental único al estilo del fondo que tiene la SUTEL o un mecanismo separado dentro de la contabilidad regulatoria, tal como lo es el recurso de la tarifa hídrica diseñado por la Empresa de Servicios Públicos de Heredia.

\subsection{Sector Transporte}

El sector transporte se ha convertido en el principal emisor de gases efecto invernadero en Costa Rica, principalmente dada su elevada dependencia de combustibles fósiles. En la actualidad, este sector consume cerca de un $78 \%$ de combustibles derivados del petróleo, demostrando así su evidente y fuerte dependencia energética de una única fuente que, en este caso, no es renovable e implica grandes impactos ambientales. Por tanto, las acciones orientadas hacia la 
reducción de las emisiones generadas en este sector tendrán un notable impacto en el cumplimiento de la meta de convertirse en un país carbono neutro adoptada por Costa Rica.

El aumento en la eficiencia de los vehículos, el aumento en la calidad de los combustibles fósiles utilizados, la incorporación de combustibles alternativos, el uso eficiente del transporte público, la construcción de ciclovías y carriles de transporte masivo son solo algunas de las medidas que ha adoptado Costa Rica como opciones de mitigación de los contaminantes generados en el sector transporte. A pesar de lo anterior, el impacto ambiental generado en este sector continúa siendo altamente relevante.

\subsubsection{Transporte público, necesidad de un cambio tecnológico}

Para el caso del transporte público en Costa Rica es evidente la necesidad de un cambio en el paradigma tecnológico si se desea avanzar hacia la meta de reducción de la gran huella de carbono que genera este sector. Según un estudio realizado por el Centro Internacional de Políticas Económicas para el Desarrollo Sostenible (Vargas, Sánchez y Otoya, 2012), se ha concluido que en materia de transporte público existe un espacio significativo para establecer procesos de cambio sustantivos en cuanto a combustibles utilizados o inclusive tecnologías.

La apuesta del país por la inclusión de nuevas tecnologías resalta como una oportunidad de desarrollo para el país. Un sector transporte bajo en emisiones sería un gran paso en el objetivo país de fomentar el uso de tecnologías verdes, sin embargo, este proceso requiere de aportes importantes en materia tarifaria y regulatoria acompañadas de medidas que garanticen una adecuada infraestructura de apoyo para el adecuado y eficiente funcionamiento del sector.

\subsubsection{Impuestos a la importación de vehículos}

Como parte de las políticas aplicadas en materia de impuestos verdes relacionadas con el sector transporte, en especial relacionado con la importación de vehículos, recientemente el Ministerio de Hacienda ha realizado un ajuste de tarifas del impuesto selectivo al consumo, en este caso para la importación de vehículos usados. 
Según el anterior Ministro de Hacienda, Edgar Ayales, Costa Rica aún mantiene una política fiscal enfocada hacia la conservación del medio ambiente y resalta que vehículos de tecnologías hibridas, eléctricas y de bajas emisiones mantienen tasas preferenciales de impuestos y cargas tributarias muy por debajo de los demás vehículos que funcionan con combustibles fósiles. Además, el país cuenta con una estructura impositiva que incrementa la carga tributaria en función de la antigüedad de los vehículos, bajo el supuesto de que un automotor más antiguo genera mayores emisiones (Ministerio de Hacienda, 2013).

Con la nueva escala de cobros, los vehículos de 0 a 6 años pagarán un impuesto selectivo al consumo correspondiente al $30 \%$ de su valor, lo que equivaldría a una carga impositiva cercana al $53 \%$. Los vehículos de más de 6 años de antigüedad tendrán que pagar un impuesto selectivo al consumo del $48 \%$ complementando así una carga impositiva total correspondiente a un $73 \%$. Además, con miras a plantear políticas de la mano con el ambiente, el Ministerio de Hacienda también decretó una reducción de 5 puntos porcentuales al impuesto selectivo a vehículos nuevos de tecnologías híbridas y eléctricas, definiendo esta tasa en un $10 \%$, la cual representa la tercera parte de la tasa aplicada a los demás vehículos. Esta reducción refleja la preocupación por disminuir el consumo de vehículos que utilicen combustibles fósiles, promoviendo una mayor importación de vehículos verdes.

De acuerdo con lo expuesto, se evidencia la preferencia del gobierno anterior de otorgar un mayor peso progresivo al transporte eléctrico, a través de incentivos fiscales a la importación. Además de las políticas fiscales anteriormente mencionadas, la creación de la Ley de Control de Emisiones Vehiculares, la ley de inspecciones del Estado Mecánico de los Vehículos, la restricción vehicular, el incentivo de la conversión de vehículos a gas LPG, las mezclas de la gasolina con etanol, entre otras, son algunas de las políticas públicas implementadas por el Estado que, de la mano con los incentivos fiscales de reducción de impuestos, procuran una de disminución de los niveles de contaminación generados por el sector transporte.

\subsubsection{Infraestructura vial}

La infraestructura vial actualmente ha sido golpeada por una oleada de fenómenos que han puesto en evidencia la necesidad de mantenimiento de estas obras. Dados los reajustes presupuestarios propuestos en periodos anteriores, se 
ha dejado de lado la importante tarea de dar un seguimiento a las obras de infraestructura nacional, principalmente las obras viales (CEPAL, 2010).

La falta de inversión pública ha desembocado en grandes problemas, los cuales se trasladan directamente a una reducción del bienestar social de la población. No cabe duda de que el cierre de vías por falta de mantenimiento causa grandes pérdidas para muchos comercios a nivel nacional. Existe un uso mayor de combustible cuando las carreteras están en mal estado, por lo que esta ineficiencia ambiental puede corregirse si se tiene un mantenimiento y reposición a tiempo.

Los congestionamientos viales provocados por las insuficientes vías alternas, además de generar ineficiencia en muchos procesos, conllevan una mayor contaminación dada la mayor concentración de vehículos. Los problemas de infraestructura vial son cada día más evidentes en Costa Rica, por lo que se pone en evidencia la necesidad de una reforma fiscal que ayude a brindar mayor presupuesto y mejores condiciones a las instituciones encargadas del mantenimiento y construcción de estas obras. Este tema es verdaderamente importante y puede también generar, mediante algún instrumento de subsidio, recursos para la movilidad alternativa como el tren.

\subsection{Sector agropecuario y ganadero}

La producción agropecuaria se ha convertido en uno de los principales generadores de emisiones de GEI, principalmente de CO2; sin embargo, su principal afectación al medio ambiente se puede asociar a la actividad ganadera, ya que el metano emitido por el ganado, resultante de un proceso digestivo, se ha convertido en uno de los principales contrincantes del medio ambiente (Ministerio de Ambiente y Energía [MINAE] e Instituto Meteorológico Nacional [IMN], 2014).

Además del metano, el óxido nitroso es otro gas distinto al CO2 y también es derivado de las actividades de carácter agropecuario. Este gas se genera principalmente de las aplicaciones de fertilizante nitrogenado que se realiza en los distintos cultivos del sector agrícola cuando se presentan algunas condiciones de 
anaerobiosis4 en el suelo. Algunos autores consideraron que la agricultura es la principal amenaza para la conservación de la biodiversidad, tanto a través de la expansión de la frontera agrícola, al talar bosques y quemar terrenos, como también al intensificar el uso de los sistemas agrícolas existentes al hacer un mayor uso de maquinas, agroquímicos, entre otros.

La sostenibilidad del sector agrícola debe incluir un uso discriminado de los factores productivos, entendiendo estos como: el terreno, los recursos naturales y la población. Esto es de particular importancia, especialmente en las regiones tropicales, donde la presión de la población, lo frágil del ambiente y la necesidad de inversiones extranjeras se combinan para poner presión sobre el medio ambiente. Brown (citado por GFA Consulting Group S.A, 2010 ) sostiene que el desarrollo sostenible de Costa Rica es una tarea difícil, dado el peso de la deuda externa sobre la economía, además del crecimiento de la esta a un ritmo que no va acorde al crecimiento demográfico. Aunado a eso, la producción de alimentos para el abastecimiento interno es insuficiente y decrece con el tiempo debido a la erosión de los suelos (GFA Consulting Group S.A, 2010).

Por otro lado, para lograr una mejora en la fertilidad de los suelos, en su mayoría se requiere cada vez más de insumos como fertilizantes. Además, ha existido una destrucción indiscriminada de bosques que ha generado una mayor emisión de carbono dada la deforestación, lo anterior aunado al uso elevado de energías fósiles en nuestra matriz energética, lo cual se complementa con la elevada y creciente densidad de población.

En Costa Rica, existe una necesidad por mejorar los niveles de competitividad del sector agrícola, esto se logra sin duda con una mejora de la infraestructura tanto física como vial, mejores puertos y aeropuertos, así como mejores servicios de telecomunicaciones. El fijar impuestos que controlen el uso de químicos, insumos que fomenten la producción orgánica, impulsos a los pequeños y medianos exportadores agrícolas, entre otras medidas, serían sin duda un avance del país en materia de integración de las políticas fiscales orientadas al sector agrícola con su preocupación por el medio ambiente.

\footnotetext{
${ }^{4}$ Capacidad que poseen algunos organismos, como hongos, bacterias, parásitos, etc., para vivir sin oxígeno molecular libre: anaerobiosis es sinónimo de vida en ausencia de oxígeno libre.
} 


\section{Las políticas a nivel local, una opción viable a futuro}

Este es un tema reciente pero importante, sobre todo por el creciente papel que tienen las municipalidades en el desarrollo local. Uno de los temas más importantes es la protección del medio ambiente y la búsqueda de espacios urbanos sostenibles, para el caso de municipalidades en la Región central. Es por eso que las iniciativas de movilidad urbana, tales como el desarrollo de ciclo-vías y de otros medios como las vías peatonales, son formas distintas de enfrentar los incentivos urbanos. Posibles alternativas en este aspecto podrían ser las paradas carbono neutral, las compras públicas municipales y el fomento al parqueo sostenible, por ejemplo con medidas especiales para vehículos eléctricos e híbridos. Las municipalidades también están a cargo del tema de desechos, tasas de recolección y otros tantos impuestos como aquellos sobre la propiedad, de los cuales podría tomarse ventaja para establecer parámetros verdes. Algunas municipalidades también tienen a cargo empresas de gestión de servicios en electricidad y agua, por lo que las políticas locales y las posibles normas o incentivos, subsidios e impuestos verdes, pueden ayudar en una mayor sostenibilidad ambiental.

\section{Conclusiones y recomendaciones}

Se puede concluir que existe un gran potencial en Costa Rica para articular la política ambiental y de cambio climático con la estructura de incentivos del sistema fiscal. Dicho cambio requiere concebirse en un entorno particular caracterizado por los aspectos enumerados a continuación. Primero, la situación deficitaria y las demandas por un Estado más comprometido con el gasto social obliga a imponer al menos de dos a tres puntos porcentuales del PIB adicionales en la carga tributaria. Segundo, dicha reforma pareciera favorecer el completar el IVA y el corregir adecuadamente el gasto tributario de cara a cumplir los objetivos de sustentabilidad ambiental. Tercero, existe un avance del lado de la demanda verde que puede completarse si se corrige la estructura de incentivos a favor de la producción y el consumo bajo en carbono y en emisiones en general.

El país ya cuenta con una gama importante de actividades dónde se ha experimentado con instrumentos y políticas para favorecer una economía verde y baja en carbono. Es importante destacar que el sector energía presenta condiciones diversas, del lado de la generación y consumo eléctrico la huella ambiental es menor 
dada la amplia base de generación con fuentes renovables. Existen oportunidades de bajar el consumo de carbono en el sector agrícola y algunas áreas del sector servicios, sin embargo, es el sector transporte donde se concentra la mayor emisión y dónde se podrían desarrollar incentivos de mayor impacto. Los impuestos a los combustibles son un ejemplo claro de uso de la renta tributaria para favorecer un ciclo de mayor sostenibilidad en el sector forestal. Sin embargo, si bien el efecto directo en reducción de demanda de combustibles es pequeño por la baja elasticidad de la demanda y la poca sustitución por lo escaso de modos de transporte, el impacto recaudatorio es amplio y el uso en protección tiene resultados muy positivos en el sector de protección de bosques.

Entre los instrumentos parafiscales y corrección de precios para favorecer el ambiente se destacan los siguientes. En primer lugar, se encuentra el impuesto directo a los combustibles, dado que ha generado mayor recaudación y un efecto directo en el fomento al PSA. En segundo lugar, las tasas ambientales tienen alguna ventaja y podrían contribuir al desarrollo de vínculos sistémicos tales como el agua ESPH y tarifa hídrica, también el caso del Volcán Poás y la tarifa de ingreso al parque. En tercer lugar, el desarrollo de mercados voluntarios ha sido escaso pero con fuerte potencial en áreas verdes, tal es el caso del PSA de hidroeléctricas y, ahora, el desarrollo del mercado local del carbono, la certificación bandera azul y el desarrollo de las empresas carbono neutral.

Para favorecer una mayor articulación de la política ambiental y de cambio climático con los instrumentos fiscales se requiere de desarrollar competencias y condiciones para conversar idiomas similares entre los distintos actores públicos, así como mejorar las competencias técnicas del Ministerio de Hacienda en la evaluación de dichos instrumentos. Es por eso que, entre otros temas, se requiere de: 1) el fortalecimiento de competencias técnicas en herramientas tales como el análisis costo beneficio con incorporación de matrices ambientales, el uso de metodologías regulatorias con implementación de tarifas verdes y el tema del análisis de escenarios con impacto de impuestos verdes son temas de gran importancia para el fortalecimiento institucional; 2) la corrección de los esquemas de gasto tributario para su alineamiento con los incentivos a una economía baja en carbono sería de gran ayuda para reducir el costo fiscal de la reforma tributaria verde; 3) capacidades de incorporar en las cuentas del Ministerio de Hacienda las fuentes de ingreso y gasto asociadas con el tema de ambiente, tanto en lo referente a prevención, mitigación y en general inversión ambiental; 4) de gran importancia 
en este tema es el tener un esquema de compras verdes que favorezca la demanda de productos y servicios desde el Estado con menor huella ambiental.

Por último, es claro que el país tiene grandes desafíos fiscales y oportunidades de integración del ambiente en este campo, pero también es de destacar que se están dando los primeros pasos en firme para crear las capacidades de articulación de lo fiscal con lo ambiental. Lo anterior es un punto central para con optimismo mirar el desarrollo de dichos instrumentos en el futuro.

\section{Bibliografía referenciada}

Barrantes, A. (2010). La Regulación del Sector Eléctrico en Costa Rica. (Documento inédito). Autoridad Reguladora de los Servicios Públicos: Costa Rica

Comisión Económica para América Latina y el Caribe [CEPAL] (2010). Boletín Infraestructuras de transporte bajas en carbono: experiencias en América Latina. Boletín Facilitación del Transporte y el Comercio en América Latina y el Caribe, 291(11). Recuperado de:

https://www.cepal.org/es/publicaciones/36173-infraestructuras-transportebajas-carbono-experiencias-america-latina

Comisión Económica para América Latina y el Caribe [CEPAL] (2016). La reforma fiscal ambiental en América Latina. Proyecto CEPAL y EUROCLIMA. Recuperado de: https://www.cepal.org/es/publicaciones/39782-la-reformafiscal-ambiental-america-latina

Decreto Ejecutivo 32868-MINAE. La Gaceta № 21 del 30 de enero del 2006. Recuperado de: http://extwprlegs1.fao.org/docs/pdf/cos67164.pdf

Estado de la Nación. (2015). Vigésimo primer Informe Estado de la Nación en Desarrollo Humano Sostenible. San José C.R: PEN.

Programa Estado de la Nación en Desarrollo Humano Sostenible (Costa Rica) (2015). Vigésimo primer Informe Estado de la Nación en Desarrollo Humano Sostenible. San José C.R: PEN.. Recuperado de: http://www.estadonacion.or.cr/21/assets/pen-21-2015-baja.pdf

Fanelli, J.M, Jiménez, J.P y López, I. (2016). La reforma fiscal ambiental en América Latina. Recuperado de: http://repositorio.cepal.org/handle/11362/39782 
Fondo Monetario Internacional (FMI). (2016). Costa Rica: Conclusiones Preliminares de la Misión de la Consulta del Artículo IV de 2016.Recuperado de:

http://www.bccr.fi.cr/publicaciones/politica monetaria inflacion/Conclusione s Preliminares mision FMI 2016 ESP.pdf

González, J. y L. Rodríguez. (2008). La reforma fiscal verde en Costa Rica. (Trabajo final de graduación de la licenciatura en Derecho). Universidad de Costa Rica. Recuperado de: http://iij.ucr.ac.cr/wp-content/uploads/bsk-pdfmanager/2017/07/La-Reforma-Fiscal-Verde-en-Costa-Rica.pdf

Jiménez, R., Amit, R. y Vindas, R. (2011). Políticas de cambio climático en Costa Rica, Integrando esfuerzos para asumir los retos.. Recuperado de: http://209.177.156.169/libreria cm/archivos/pdf 709.pdf

Acquatella, J. y Gómez, J. (2005). Política Fiscal y Medio Ambiente: bases para una agenda común. Recuperado de:

https://www.cepal.org/es/publicaciones/2430-politica-fiscal-medio-ambientebases-agenda-comun

Ministerio de Planificación y Política Económica [MIDEPLAN]. (2010). Plan Nacional de Desarrollo 2011-2014 "Maria Teresa Obregón Zamora". San José, Costa Rica: MIDEPLAN. Recuperado de: https://documentos.mideplan.go.cr/alfresco/d/d/workspace/SpacesStore/122 fcd1c-53a7-47a7-a0ad-84cac6f1d7b9/PND-2011-2014-Maria-TeresaObregon-Zam

Ministerio de Planificación y Política Económica [MIDEPLAN]. (2015). Plan Nacional de Desarrollo 2015-2018 "Alberto Cañas Escalante". San José, Costa Rica: MIDEPLAN. Recuperado de: https://documentos.mideplan.go.cr/alfresco/d/d/workspace/SpacesStore/cd1 da1b4-868b-4f6f-bdf8-b2dee0525b76/PND\%2020152018\%20Alberto\%20Ca\%C3\%B1as\%20Escalante\%20WEB.pdf

Ministerio de Ambiente y Energía [MINAE] e Instituto Meteorológico Nacional [IMN] (2014). Tercera Comunicación Nacional del Marco de las Naciones Unidas sobre Cambio Climático. Recuperado de:

http://www.nacion.com/vivir/ambiente/Tercera-Comunicacion-NacionalConvencion-Climatico LNCFIL20141124 0001.pdf

Ministerio de Hacienda. (2013). En ruta hacia la consolidación fiscal: agenda para un diálogo nacional. Recuperado en:

http://www.hacienda.go.cr/docs/527b8da0d2859 documento final.pdf 
Ministerio de Hacienda. (2016). El presupuesto nacional en Costa Rica, 2015. Recuperado de:

http://www.hacienda.go.cr/docs/5405f856eea45 Folleto\%20Presupuesto\%2 0Nacional\%202015a.pdf

Organisation for Economic Co-operation and Development (2016). Estudio económico de la OCDE Costa Rica. Recuperado de: http://www.oecd.org/eco/surveys/economicsurvey-costa-rica.htm

Orozco J., y Ruiz, K. (2001). Uso de Instrumentos Económicos para la Gestión Ambiental en Costa Rica. Serie Medio Ambiente y Desarrollo, 41. Recuperado de: http://repositorio.cepal.org/handle/11362/5750

Paniagua, F. (2016). El proceso de elaboración de la contribución nacional INDC de Costa Rica. Recuperado de: http://ledslac.org/wpcontent/uploads/2016/04/estudio de caso indc costa rica 30 marzo publ icado-3.pdf

PNUMA-GEO-5. (2012). Perspectivas del Medio Ambiente Mundial. Medio ambiente para el futuro que queremos. Recuperado de: https://www.cbd.int/doc/meetings/nr/rw5nr-la-01/other/rw5nr-la-01-pnumaes.pdf

Reino Unido. Department for International Development, SIECA, DANIDA, NU. CEPAL (2012). La economía del cambio climático en Centroamérica. Síntesis 2012. Recuperado de: https://www.cepal.org/es/publicaciones/26058-la-economia-cambioclimatico-centroamerica-reporte-tecnico-2011

Rodríguez, C. y Echeverría, J. (enero del 2002). Herramientas Económicas y Fiscales para la Gestión Ambiental en Costa Rica. XV Seminario Regional de Política Fiscal. Seminario llevado a cabo en Santiago, Chile. Recuperado: https://www.cepal.org/ilpes/noticias/paginas/1/35691/JA Costa Rica PSA corto.pdf

Segura, O. (2000). Sustainable systems of innovation: the forest sector in Central America. Aalborg: Aalborg Universitetsforlag.

Vargas, L., Sánchez, R. y Otoya, M. (2012). Transporte Público y Cambio Climático. El caso de Taxis y Autobuses de Servicio Público. Proyecto Apoyo a la Preparación de Estrategias de Desarrollo bajo en emisiones y adaptado al cambio climático (Documento inédito) 